\title{
Knowledge, attitude, and practice of gynecologists' regarding Hepatitis B in pregnant females in Jordan
} \author{
AlNajjar $^{2}$ \\ ${ }^{1}$ Applied Science Private University Faculty of Pharmacy \\ ${ }^{2}$ Applied Science Private University \\ ${ }^{3}$ The Hashemite University Faculty of Medicine
}

Bayan Othman ${ }^{1}$, Raja'a Alqudah ${ }^{2}$, Iman Basheti ${ }^{2}$, Hamza Omoush ${ }^{3}$, and Mohammad

April 28, 2020

\begin{abstract}
Early detection of Hepatitis B virus (HBV) in pregnant females starts by a request of the gynecologists, which is based on their knowledge and awareness of the guidelines on conducting these tests. This is an observational cross-sectional study that investigated the gynecologists' knowledge, attitude and practice regarding HBV during pregnancy across Jordan. A random sample of 150 gynecologists were approached, from 3 major cities in Jordan, and asked to fill a questionnaire that assessed their knowledge, attitude and practice. Statistical analysis was conducted using SPSS. One hundred and seven gynecologists have participated in the study. Most of the respondents were females, residents, and less than thirty years old. Although $97.2 \%$ of the practitioners agreed on the importance of the pregnant females on HBV, only $43 \%$ were aware of the obligatory protocol in Jordan regarding HBV screening, and only $55 \%$ would screen the pregnant females to HBV in-practice. Significant association was found between screening rates to HBV and both, level of specialty and experience of practitioners. Approximately $60 \%$ of practitioners were aware of HBV perinatal transmission risk. Only $19.6 \%$ always referred the infected pregnant females to other specialists regardless of the viral load. While $47.7 \%$ of practitioners agreed on recommending antiviral therapy for third trimester pregnant females, only $12.1 \%$ would always/often prescribe them. A noteworthy lack of Hepatitis B knowledge and screening practice among gynecologists in Jordan have been observed. A national program designed to increase the awareness of HBV testing in Jordan for both patients and gynecologists is called for.
\end{abstract}

\section{Author Contributions}

- Study concept and design: MAN, IB, HO.

- Acquisition of data: BO.

- Analysis and interpretation of data: BO, RQ, IB, MAN.

- Drafting of the manuscript: BO, RQ, MAN.

- Critical revision of the manuscript for important intellectual content: BO, RQ, MAN, IB.

- All authors had access to the study data and reviewed and approved the final manuscript.

Agreement to be accountable for all aspects of the work in ensuring that questions related to the accuracy or integrity of any part of the article are appropriately investigated and resolved: all authors.

\section{Introduction}

Hepatitis B is a viral infection caused by DNA-viruses called Hepatitis B Virus (HBV), belonging to the family Hepadnaviridae. Normally, the virus attacks the liver causing both acute and chronic disease conditions ${ }^{1}$. The World Health Organization (WHO) highlights HBV as a major global health problem, and the most 
serious type of viral hepatitis. The HBV is highly contagious and can be transmitted by percutaneous or mucosal exposure to infected blood and various body fluids, as well as through saliva, menstrual, vaginal, and seminal fluids ${ }^{1}$. Based on the WHO estimation, about 780,000 people die each year because of Hepatitis $\mathrm{B}$, if not treated well at an early stage ${ }^{2}$. Those elevated numbers highlight the fact that Hepatitis $\mathrm{B}$ is a dangerous infectious disease that should be kept under control.

The Ministry of Health in Jordan has a clear protocol about HBV, which states that Hepatitis B tests should be done during pregnancy for all females ${ }^{3}$. Vaccination against Hepatitis B has been mandatory to all newborns since 1995. In Jordan, a study by Batayneh and Bdour stated that about $86 \%$ of the pregnant females are susceptible to HBV infection, $10 \%$ have been previously infected, $3 \%$ had acute HBV infection and $1.5 \%$ had a chronic infection ${ }^{4}$. Apart from few prevalence studies conducted in Jordan ${ }^{5}$ and the Middle East ${ }^{6}$, there have been no previous studies done to assess the knowledge, attitude and practice of gynecologists regarding Hepatitis B testing, specifically for pregnant females. This study is considered to be a pioneer in this area as it is aimed to provide vital information on the medical practice in Jordan regarding Hepatitis B screening for pregnant females.

Hence, the current study aims to assess gynecologists' knowledge, attitude and practice regarding HBV screening on pregnant females in Jordan.

\section{Methods}

\section{Study Setting}

This cross-sectional study involved gynecologists practicing in three major cities of Jordan: Amman (the capital), Irbid and Al Zarqa (two urban cities) relying on the population density and the geographical distribution. Irbid is located to the north of Amman and it is the second largest city in Jordan by population after Amman (according to the Department of Statistics in Jordan) ${ }^{7}$. Irbid is surrounded by a large number of villages that lack specialized hospitals; therefore, patients have to come to Irbid for their medications. Al Zarqa is located in the northeast of Jordan and is considered to be the largest city after Amman (area-wise) and the third largest city by population in Jordan.

\section{Survey development}

The gynecologist's questionnaire (Appendix 1) was designed critically and concisely based on literature surveys of similar studies and the general principles of good survey design ${ }^{8-11}$. The survey contained close-ended and open-ended questions that can be completed within an average of 15 minutes. To ensure comprehension, clarity, readability and acceptability, the survey was piloted by a group of volunteer gynecologists $(\mathrm{n}=10)$ during a pilot study that was conducted over a month period. Refinements were made as needed before the last version of the survey was finalized.

The survey consisted of four main parts; the first part was designed to collect data on the gynecologists' demographic characteristics including age, gender, years in practice, and level of specialty. The second part was designed to assess gynecologists' knowledge regarding HBV in pregnancy. The third part was designed to evaluate gynecologists' attitude regarding HBV testing including their attitude regarding the importance of screening pregnant females on HBV and counseling them about it. The last part was designed to assess gynecologists' practice regarding HBV testing during female pregnancy. The questionnaire included Likert scale questions that measured their basic knowledge and daily practice regarding Hepatitis B.

\section{Study participants}

A convenience sample of 150 Gynecologists were approached to participate in the study. In Amman ( $\mathrm{n}=44)$, Al Zarqa $(\mathrm{n}=32)$ and Irbid ( $\mathrm{n}=31)$ gynecologists were recruited into the study. Gynecologists' specialists and residents were randomly visited during the last quarter of 2018 (from October to December), practicing in private and public healthcare facilities. They were approached in the clinics by one of the research team and provided with an information leaflet about the study. Recruited gynecologists provided consent before 
study participation. A written copy of the survey was then handed over to be completed anonymously and returned to the researcher in sealed envelopes.

\section{Data analysis}

Collected data was coded and entered using the Statistical Package for Social Science (SPSS) version 24 (Chicago, Illinois). Descriptive statistics (percentages and frequencies) were computed for all study variables. The proportion of participants answering the different questions in the survey were compared using Pearson's $\chi 2$ test. Correlation testing exploring associations between socio-demographic data and gynecologists' practice characteristics were identified using Pearson's correlation and chi-square test for significance. A probability value of $<0.05$ was considered to be statistically significant for all analyses tests.

\section{Ethical approval}

Ethical approval was obtained from the Jordanian Ministry of health (Approval number: IRB/12617). As well as a second approval was taken for this study from the Ethical Research Board at the Applied Science Private University (Approval number: 2018-PHA-1).

\section{Results}

\section{Demographic characteristics}

A total of 107 Gynecologists across Jordan participated in the current study. The majority of practitioners were females $(\mathrm{n}=84)$ representing $78.5 \%$ of the study sample compared to $21.5 \%$ males $(\mathrm{n}=23)$. While the minority of the participants included specialists $(\mathrm{n}=16,15 \%)$, most of the participated gynecologists were residents $(\mathrm{n}=91,85 \%)$ from different age groups, with variations in the level of residency and years in practice (Table 1). The responding participants were recruited from public $(\mathrm{n}=60,56.1 \%)$ and private $(\mathrm{n}=47,43.9 \%)$ healthcare facilities. Participants practiced in Amman $(\mathrm{n}=44,41 \%)$, while the rest practiced in Al Zarqa $(\mathrm{n}=32,30 \%)$ and Irbid $(\mathrm{n}=31,29 \%)$. Most of the surveyed residents were in their fourth year $(\mathrm{n}=24,26.4 \%)$ and the first year $(\mathrm{n}=23,25.3 \%)$ of residency.

\section{Knowledge of gynecologists}

The majority of participants agreed on the importance of screening pregnant females on HBV during their first prenatal visit $(n=95,88.8 \%)$. However more than half of them $(n=61,57.0 \%)$ did not know that there is an obligatory protocol in Jordan demands the screening of pregnant females on HBV.

Most of the participants $(\mathrm{n}=89,83.2 \%)$ agreed on referring pregnant females to a specialist if they were found to have a positive HBeAg result. However, only $57.0 \%(\mathrm{n}=61)$ of them were familiar with the HBV DNA viral load that is required for referral to take place. Only $60.7 \%(\mathrm{n}=65)$ of the practitioners were aware of the risk of perinatal transmission of HBV infection when the infant comes into contact with vaginal blood and secretions during delivery. Almost half of the practitioners believed that antiviral medications generally are not recommended for HBV during pregnancy $(\mathrm{n}=49,45.8 \%)$.

Only $47.7 \%(\mathrm{n}=51)$ agreed on giving antiviral therapy for third trimester pregnant females with high viral loads ( $>1$ million copies $/ \mathrm{mL}$ or $200,000 \mathrm{IU} / \mathrm{mL}$ ). There was a significant difference between rate of practitioners in public hospitals who agreed on recommending antiviral agents for third trimester pregnant females with high viral loads and practitioners in private hospitals ( $\mathrm{p}=0.042$; Fig. 1).

A minority $(29.9 \%, \mathrm{n}=32)$ agreed on prescribing tenofovir as the first line treatment for pregnant females with HBV, while $(46.7 \%, \mathrm{n}=50)$ of the surveyed participants were undecided.

Furthermore, $69.2 \%(\mathrm{n}=74)$ of participants disagreed on selecting c-section as the mode of delivery in the setting of maternal HBV, and $71 \%(n=76)$ agreed that carrier mothers of Hepatitis B can breastfeed their babies.

\section{Attitude of gynecologists}


Almost all participated practitioners had positive response towards the importance of HBV tests. Majority of them agreed on the importance of counseling the pregnant females about Hepatitis B screening (97.2\%, $\mathrm{n}=104)$. In addition, $94.4 \%(\mathrm{n}=101)$ believed that pregnant females have to be screened for HBV. About $90 \%$ $(\mathrm{n}=96)$ showed conformity on the importance of having a HBV screening protocol that should be followed for pregnant females.

\section{Practice of gynecologists}

Only $55 \%(\mathrm{n}=55)$ of participants reported screening pregnant females on HBV during their current daily practice. A significant difference was found between the screening rates of HBV and both; level of specialty and experience of the practitioners respectively $(\mathrm{p}=0.002, \mathrm{p}=0.044$; Fig. 2), as longer years of experience associated with more screening conducted.

Binary logistic regression modeling (Nagelkerke $\mathrm{R}$ Square $=0.145, \mathrm{p}=0.318$ ) showed no significant association between screening rates of Hepatitis B (dependent variable) and the independent variables: years since graduation and level of specialty (Table 2).

As for the practice settings, there was a significant difference between the level of specialty and the type of health facility where the doctors practiced ( $\mathrm{p}=0.007$; Fig. 3). Most of the surveyed specialists practiced in private hospitals $(\mathrm{n}=12)$, while most of the residents practiced in public educational hospitals $(\mathrm{n}=56)$. Moreover, it was found that screening rates of pregnant females on HBV were higher in private hospitals than public hospitals but were not statistically significant ( $p=0.136$; Fig. 3 ).

Most of the practitioners $(\mathrm{n}=61,59.2 \%)$ relied on HBsAg level as the first marker for infection followed by HBeAg levels $(n=8,7.8 \%)$. Few participants $(n=20,19.6 \%)$ always referred HBsAg positive pregnant females regardless of HBV DNA viral load, while $39.2 \%(\mathrm{n}=40)$ were uncertain; they would sometimes refer them. Almost half of the surveyed practitioners $(n=42,42.4 \%)$ never prescribe antiviral therapy for pregnant females in the third trimester, only $12.1 \%(\mathrm{n}=12)$ answered always/very often they would when needed. Cesarean section recommendation as a mode of delivery in HBV pregnant females was not common between the practitioners, as $44.4 \%(\mathrm{n}=44)$ would never recommend it, $18.2 \%(\mathrm{n}=18)$ stated that they would rarely recommend it and $23.2 \%(\mathrm{n}=23)$ would sometimes. More than half of practitioners $(\mathrm{n}=61,61 \%)$ would always recommend Hepatitis B immunoglobulin (HBIG) injection at birth for newborns of HBsAg carrier mothers as well as $51 \%(\mathrm{n}=49)$ would always request HBIG administration to newborns in their practicing hospital. Part of the practitioners $(\mathrm{n}=54,54.6 \%)$ reported always/very often following up with infants born to HBsAg carrier mothers. When the practitioners were asked about the greatest barriers against HBV screening in early pregnancy, $64.5 \%$ of them $(n=69)$ referred that the lack of patient's education, followed by the high cost of the test $(\mathrm{n}=55,51.4 \%)$, as the greatest barriers (Fig. 4$)$.

\section{Discussion}

It is very important to screen pregnant females for Hepatitis B infection on the first perinatal visit ${ }^{12}$. In any healthcare system, priorities, screening requirements and medical procedures are governed by a clear, published protocol by the health governing official bodies, mainly the Ministry of Health. Although (97.2\%) of practitioners in Jordan agreed on the importance of counseling and screening the pregnant females on HBV, knowledge among them was limited.

More than half of the surveyed practitioners were not aware of the presence of the obligatory protocol for Hepatitis B screening in Jordan. Only $55 \%$ offered screening for Hepatitis B on the first perinatal visit during their daily practice. This is most likely due to lack of awareness and knowledge of the practitioners on the importance of Hepatitis B screening. Also, the unclear policies of practice that are followed in the healthcare facilities may contribute to such result.

It has been reported that healthcare workers at the Jordanian hospitals do not have enough trainings, nor a clear protocol on how to deal with infectious diseases including Hepatitis B ${ }^{13}$. In Jordan the vaccination against Hepatitis B has been mandatory for all newborns since $1995^{3}$. This fact may also have allowed the practitioners to ignore the screening of females who were born before 1995, assuming that there is no need to 
do the tests. However, a large proportion of those females are still in the childbearing age. Another reason could be the lack of practitioners adherence to the protocols and safety precautions, although they would have known about them ${ }^{14}$.

This study identified a clear association between the specialty of the practitioner and offering the screening for Hepatitis B $(p=0.002)$. In addition, there was a clear association between the years of practice of the practitioner and the screening rates of pregnant females on Hepatitis B in daily practice $(\mathrm{p}=.044)$. Most of the surveyed specialists screen pregnant females for Hepatitis B on their first perinatal visit in contrary to practicing residents. This result may be attributed to the fact that practicing specialists have more years in practice and experience compared to practicing residents, resulting in higher screening rates. As well as the association between the type of health facility and the level of specialty $(\mathrm{p}=0.007)$ adds to this result; most of the practicing specialists work in private hospitals, where most of their patients are in a better educational and financial state compared to public hospitals' patients. Most of these patients are willing to have the HBV test performed regardless of the cost barrier. All of the above contributes to have higher screening rates in private hospitals compared to public hospitals. Interestingly an earlier study in Italy observed similar results; it was reported that the risk of not screening the pregnant women for Hepatitis B was also found to be higher in public hospitals compared to private hospitals ${ }^{15}$.

The maternal viral load plays a crucial role in determining the vertical transmission rate to infants ${ }^{16,17}$. High viral loads of $>108 \mathrm{IU} / \mathrm{mL}$ were associated with higher risk of transmission, as well as with higher probability of failure to prophylactic immunization ${ }^{16,18,19}$. Thus, the 2018 American Association for the Study of Liver Diseases (AASLD) guidelines recommends antiviral therapy for pregnant women with HBV DNA level $>200,000 \mathrm{IU} / \mathrm{mL}$. The AASLD guidelines recommends tenofovir as one of the preferred antiviral medications due to its safety and lack of resistance concerns ${ }^{20}$. It is reported that receiving antiviral therapy on the third trimester of pregnancy reduces the risk of mother-to-child transmission (MTCT) massively ${ }^{21}$. According to the results there was a significant difference between the type of healthcare facility and the recommendation of antiviral therapy to third trimester pregnant females with high viral loads $(>1$ million copies $/ \mathrm{mL}$ or $200,000 \mathrm{IU} / \mathrm{mL}$ ). More than half of the public hospitals' practitioners (58.3\%) agreed on giving antiviral agents when needed in contrary to private hospitals practitioners (34\%). This may be due to the fact that practitioners in public hospitals are mostly residents who are still training and continuously being reminded and updated on the protocols of treatment, in contrary to specialists who mostly practice in their private gynecological clinics in private hospitals.

Data regarding the safety of taking antiviral therapies during pregnancy or breastfeeding has not been reported yet, as there are insufficient long term safety studies on infants ${ }^{20}$. The AASLD (2018) guidelines reported that breastfeeding is not contraindicated during therapy because the excreted levels of antiviral agents in breast milk are insignificant ${ }^{20}$. Adding on, numerous studies have been reporting the same findings on low levels of antiviral agents in breast milk ${ }^{22}$. As long as there is immunization and vaccination programs followed, it is reported to have no risk of Hepatitis B transmission to breast fed infants ${ }^{23}$. According to our results, this has been confirmed by most of the surveyed practitioners, as $71 \%$ of them approved breast feeding for carrier mothers and $70 \%$ recommended Hepatitis B immunoglobulin (HBIG) injection at the birth for newborns of HBsAg carrier mothers.

The mode of delivery has not been proved to affect the risk of Hepatitis B transmission to the infant ${ }^{20}$. The AASLD (2018) guidelines do not recommend a C-section to be performed to the pregnant females as there is no clear benefit found compared to other modes of delivery ${ }^{20}$. A previous research study reported that there is no significant effect for rupture of membranes and labor on vertical transmission of Hepatitis B ${ }^{24}$. In agreement, $62.6 \%$ of our surveyed participants did not recommend a C-section for HBV pregnant females.

\section{Conclusion}

According to the study results, although most of participated gynecologists agreed on the importance of screening and counseling the pregnant women on HBV, there is a huge lack of knowledge and practice with regards to screening and treatment provision of HBV among gynecologists in Jordan. The ministry of 
health in Jordan plays a crucial role in highlighting the mandatory protocol of screening pregnant females on Hepatitis B. Training programs and close monitoring of practitioners in public and private healthcare facilities are recommended. As well as, there is a huge need to raise the awareness of the practitioners about the risk of exposure to Hepatitis B during their daily practice, as most of them were found unaware of the risk of transmission. All of the efforts should be gathered from the different healthcare professionals to halt the spreading of this major global health problem, reducing the risk of vertical transmission of Hepatitis B.

\section{Limitations}

Many gynecologists working in public hospitals refused to participate in the survey due to lack of time, hence, most of the surveyed practitioners are residents and are still under training in hospitals. Equality in ratio between specialists and residents are recommended for future studies to give a more accurate result reflecting the situation with regards to HBV screening in the different healthcare facilities.

Acknowledgments: The authors are grateful to the Applied Science Private University Amman, Jordan, for the support granted to this research project. The authors wish to thank the healthcare professionals who participated in this research study. The authors thank also the Ministry of Health in Jordan for giving the permission to visit their health facilities and collect data.

Funding: This research did not receive any specific grant from funding agencies in the public, commercial, or not-for-profit sectors.

Conflicts of Interest: The authors declare no financial or other potential conflicts of interest exist.

Data Availability: The datasets generated during and/or analyzed during the current study are available from the corresponding author on reasonable request.

\section{References}

1. Korsman SNJ, van Zyl GU, Nutt L, Andersson MI, Preiser W. Hepadnaviruses. In: Korsman SNJ, van Zyl GU, Nutt L, Andersson MI, Preiser W, eds. Virology . Edinburgh: Churchill Livingstone; 2012:70-71.

2. Organization WH. Global Hepatitis Report 2017. Geneva2017.

3. Hamoudi W, Qaseer B, Abdallat M, et al. Jordan viral hepatitis B \& C 2016. 2016.

4. Batayneh N, Bdour S. Risk of Perinatal Transmission of Hepatitis B Virus in Jordan. Infectious Diseases in Obstetrics and Gynecology. 2002;10(3).

5. Bank-Jordan NB. National Blood bank data Jordan2015.

6. Al Awaidy S, Abu-Elyazeed R, Al Hosani H, et al. Sero-epidemiology of hepatitis B infection in pregnant women in Oman, Qatar and the United Arab Emirates. J Infect. 2006;52(3):202-206.

7. Statistics. Do. Population of Hashemite Kingdom of Jordan According to the 1952, 1961, 1979 ,1994,2015 and 2018 Censuses. . 2018.

8. Adekanle O, Ndububa DA, Olowookere SA, Ijarotimi O, Ijadunola KT. Knowledge of hepatitis B virus infection, immunization with hepatitis $\mathrm{B}$ vaccine, risk perception, and challenges to control hepatitis among hospital workers in a Nigerian tertiary hospital. Hepatitis research and treatment. 2015;2015.

9. Hu Y, Dai X, Zhou Y-H, Yang H. A knowledge survey of obstetrics and gynecology staff on the prevention of mother-to-child transmission of hepatitis B virus. The Journal of Infection in Developing Countries. 2013;7(05):391-397.

10. Niu B, Halegoua-De Marzio D, Fenkel JM, Herrine SK. Obstetricians' and gynecologists' knowledge, education, and practices regarding chronic hepatitis B in pregnancy. Annals of gastroenterology.2017;30(6):670.

11. Boynton PM, Greenhalgh T. Selecting, designing, and developing your questionnaire. BMJ. 2004;328(7451):13121315 . 
12. Force UPST. Screening for Hepatitis B Virus Infection in Pregnant Women: US Preventive Services Task Force Reaffirmation Recommendation StatementUSPSTF Recommendation: Screening for Hepatitis B Virus Infection in Pregnant WomenUSPSTF Recommendation: Screening for Hepatitis B Virus Infection in Pregnant Women. JAMA.2019;322(4):349-354.

13. Hassan Z, Wahsheh M, Shishani K, Pryor E. Hepatitis needs assessment among Jordanian healthcare workers. International nursing review.2008;55(2):142-147.

14. Chogle N, Chogle M, Divatia J, Dasgupta D. Awareness of post-exposure prophylaxis guidelines against occupational exposure to HIV in a Mumbai hospital. National Medical Journal of India.2002;15(2):69-71.

15. Spada E, Tosti ME, Zuccaro O, Stroffolini T, Mele A, Group CS. Evaluation of the compliance with the protocol for preventing perinatal hepatitis B infection in Italy. Journal of Infection.2011;62(2):165-171.

16. Singh AE, Plitt SS, Osiowy C, et al. Factors associated with vaccine failure and vertical transmission of hepatitis B among a cohort of Canadian mothers and infants. J Viral Hepat. 2011;18(7):468-473.

17. Wiseman E, Fraser MA, Holden S, et al. Perinatal transmission of hepatitis B virus: an Australian experience. Medical Journal of Australia. 2009;190(9):489.

18. Wen WH, Chang MH, Zhao LL, et al. Mother-to-infant transmission of hepatitis B virus infection: significance of maternal viral load and strategies for intervention. J Hepatol. 2013;59(1):24-30.

19. Zou H, Chen Y, Duan Z, Zhang H, Pan C. Virologic factors associated with failure to passive-active immunoprophylaxis in infants born to HBsAg-positive mothers. J Viral Hepat. 2012;19(2):e18-25.

20. Terrault NA, Lok AS, McMahon BJ, et al. Update on prevention, diagnosis, and treatment of chronic hepatitis B: AASLD 2018 hepatitis B guidance. Hepatology. 2018;67(4):1560-1599.

21. Seo KI, Bae SH, Sung PS, et al. Effect of antiviral therapy in reducing perinatal transmission of hepatitis B virus and maternal outcomes after discontinuing them. Clinical and molecular hepatology. 2018;24(4):374.

22. Benaboud S, Pruvost A, Coffie PA, et al. Concentrations of tenofovir and emtricitabine in breast milk of HIV-1-infected women in Abidjan, Cote d'Ivoire, in the ANRS 12109 TEmAA Study, Step 2.Antimicrobial agents and chemotherapy. 2011;55(3):1315-1317.

23. Hill JB, Sheffield JS, Kim MJ, Alexander JM, Sercely B, Wendel Jr GD. Risk of hepatitis B transmission in breast-fed infants of chronic hepatitis B carriers. Obstetrics $\mathcal{E}$ Gynecology.2002;99(6):1049-1052.

24. Cheung KW, Seto MTY, So PL, et al. The effect of rupture of membranes and labour on the risk of hepatitis B vertical transmission: Prospective multicentre observational study. European Journal of Obstetrics 83 Gynecology and Reproductive Biology. 2019;232:97-100.

Figure legends

Figure 1. Practitioners response $(\mathrm{n}=107)$ to "Third-trimester antiviral therapy is recommended for females with high viral loads (>1 million copies/mL or 200,000 IU/mL)".

*Pearson's chi -square test.

Figure 2. Associations between the level of specialty and experience of practitioners $(n=100)$ with the screening rates of pregnant females on Hepatitis B during practice.

*Pearson's chi -square test.

Figure 3. Proportions of practitioners $(\mathrm{n}=107)$ and screening rates of $\mathrm{HBV}$ for pregnant females in private and public hospitals.

*Pearson's chi -square test. 
Figure 4. Barriers associated with HBV testing in Jordan based on reported practitioners' perspectives ( $\mathrm{n}=$ 107).

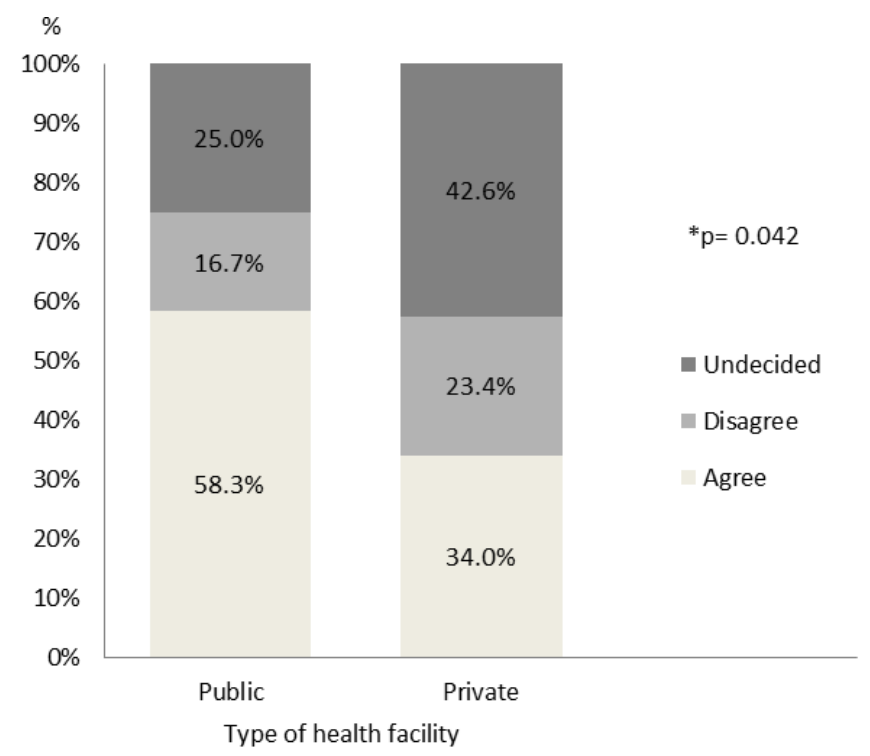

Figure 1. Practitioners response ( $\mathrm{n}=107)$ to "Third-trimester antiviral therapy is recommended for females with high viral loads ( $>1$ million copies $/ \mathrm{mL}$ or 200,000 $\mathrm{IU} / \mathrm{mL}) "$.

*Pearson's chi -square test.

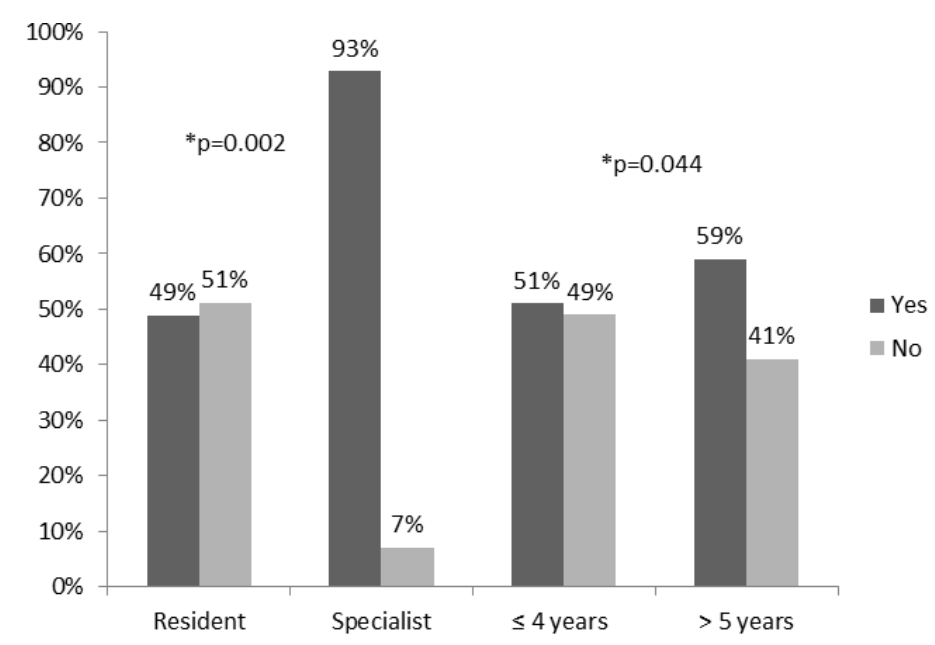

Figure 2. Associations between the level of specialty and experience of practitioners $(\mathrm{n}=100)$ with the screening rates of pregnant females on Hepatitis B during practice. *Pearson's chi -square test. 


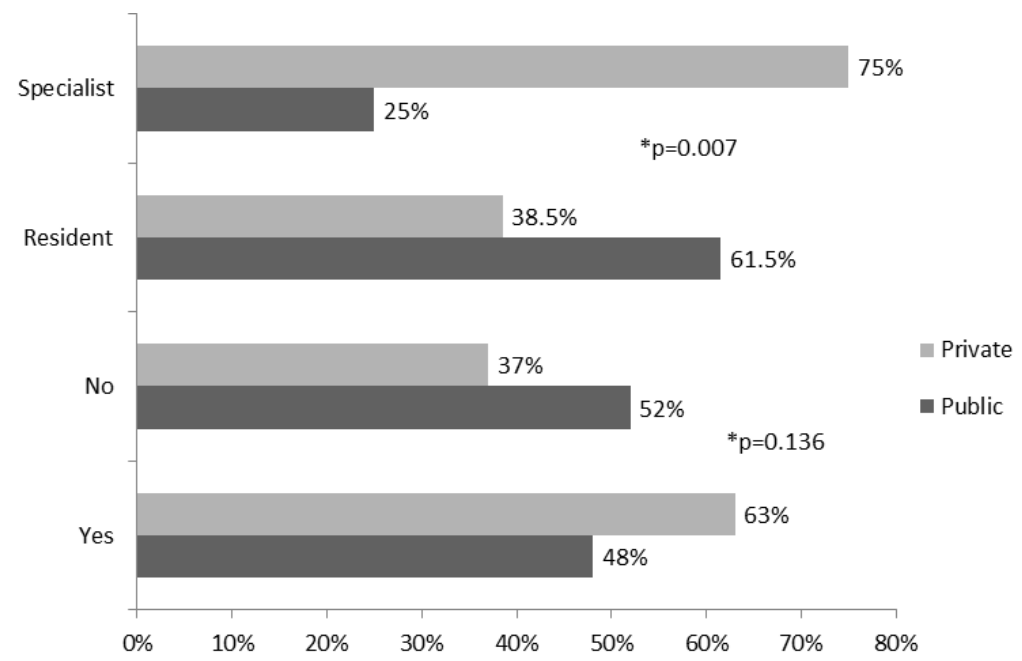

Figure 3. Proportions of practitioners $(n=107)$ and screening rates of $\mathrm{HBV}$ for pregnant females in private and public hospitals.

*Pearson's chi -square test.

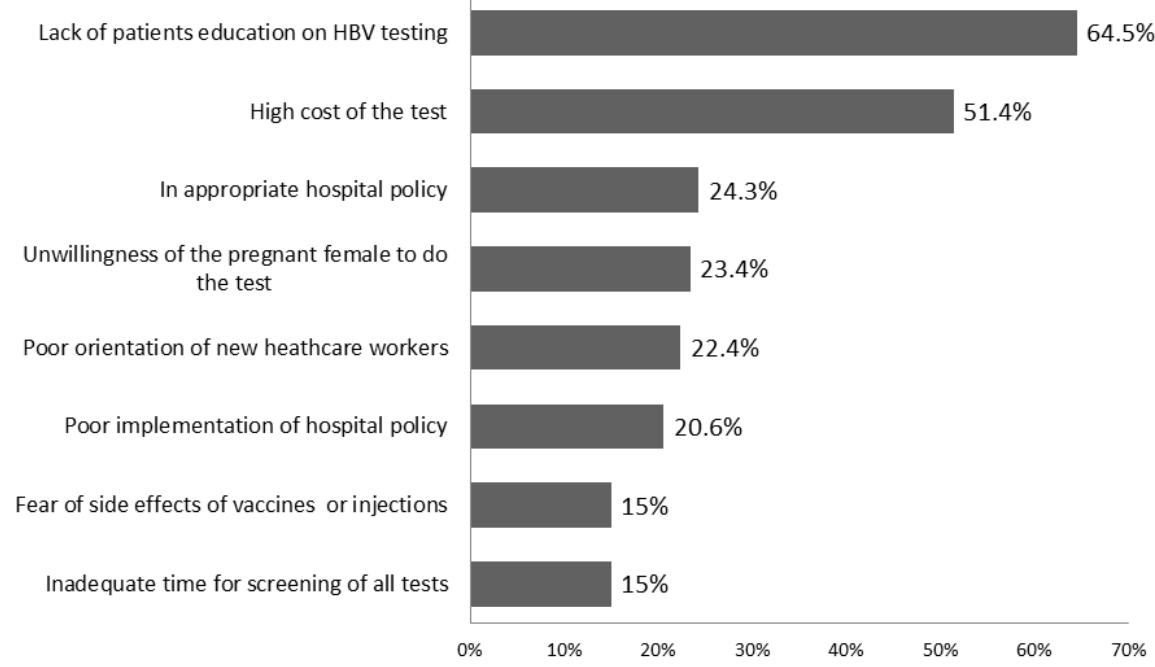

Figure 4. Barriers associated with HBV testing in Jordan based on reported practitioners' perspectives $(\mathrm{n}=107)$.

\section{Hosted file}

Tables.docx available at https://authorea.com/users/310287/articles/441112-knowledge-attitudeand-practice-of-gynecologists-regarding-hepatitis-b-in-pregnant-females-in-jordan 